\title{
Case study of caylee anthony thru the forensics investigation
}

\author{
Abstract \\ July 16, 2008 was just another day as a Crime Scene Investigator for the Orange County \\ Sheriff's Office in Orlando, Florida. This day is different, yet the same, as a beautiful girl \\ has gone missing. Her name, Caylee Marie Anthony. \\ Keywords: national center of missing child \& abc news report "missing children in amer- \\ ica unsolved cases" may 7,2013 , florida department of children and families, photo of vehi- \\ cle taken by csi gerardo bloise (orange county sheriff's office), cornell university law school
}

Volume 4 Issue 4 - 2017

\section{Gerardo Bloise}

GJ Forensics LLC, USA

Correspondence: Gerardo Bloise, MPA, CCSI, G\&J Forensics PO Box 324, Oakland, Florida, USA, Tel 34760 (407) 608-3066; Email gjforensicsllc@gmail.com

Received: March II, 2017 | Published: April 24, 2017

\section{Introduction}

On July 16, 2008 at approximately 7:46 p.m. Detective Charity Beasley arrived at the Forensics Unit garage area, where a vehicle had been impounded from the missing child's family home, as the information provided was that it was still being investigated for that of a missing child. Challenging, is an understatement in relating how this case was viewed from a forensic perspective, due to it gravitating from a missing child to a homicide. There is a report that informed that about 800,000 children are reported missing each year in the United States, and broken down makes it about 2,000 per day and that number increases every year. ${ }^{1}$

There is list 203 missing children in Florida alone. This is a permanent and social problem without counting the unsolved homicides related to missing child cases. ${ }^{2}$ Why is this information is important? If you recall, the Caylee Anthony case at first glance was just that, a missing child. Yet there was a time when the question then became, "is it a missing child, or a homicide?" And the only way to answer the question is through a thoroughly executed forensics investigation.

\section{The vehicle}

In regards to Casey Anthony's vehicle, a 1998 White Pontiac Sunfireb $^{3}$ was the only piece of evidence I received.

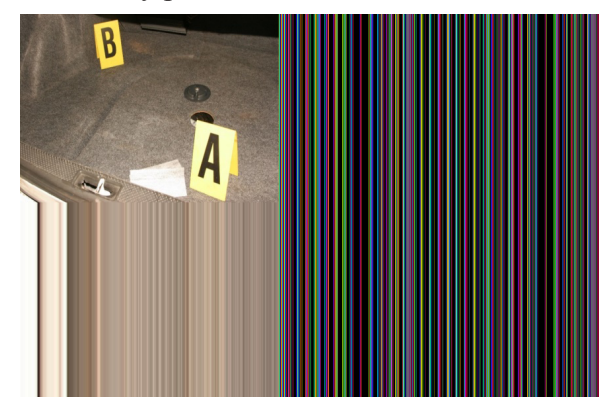

1National Center of Missing Child \& ABC News report "Missing Children in America Unsolved Cases" May 7,2013

${ }^{2}$ Florida Department of Children and Families.

${ }^{3}$ Photo of vehicle taken by CSI Gerardo Bloise (Orange County Sheriff's Office).
Initially, it was a difficult case for me, processing a vehicle with no information other than the vehicle being part of an on-going investigation regarding a missing child.

\section{Visual inspection}

However, the same procedures must be followed when dealing with forensic evidence. The first step is to document the evidence received as you receive it, and so I photographed the exterior and interior of the vehicle. Once I have photographed the vehicle, I begin to inspect it in the same manner, from the outside, in. On the surface, the vehicle looks clean. I mean, if you looked at the vehicle from the outside, it looked clean. Then I opened the door. There is an odor of death, a certain smell, and once you experience it, you will never forget it. And if you smell it again, you'll know exactly what the smell is associated to, and that familiar foul odor is one associated with human decomposition. What does that mean to me from a forensic point of view? At some point there was a dead body in this car. How do I come to that conclusion? Easy After 28 years in law enforcement, both here and in Puerto Rico, the majority of my cases dealt with dead bodies, and bodies already in full decomposition. And as I stated earlier, once you experience the odor of a human body decomposing, it is one you will never forget. The photographs of the interior of the vehicle indicate a relatively clean car (see below). Knowing what my experience tells me may have been in this vehicle, I begin to utilize the equipment necessary to detect what the human eye cannot see.

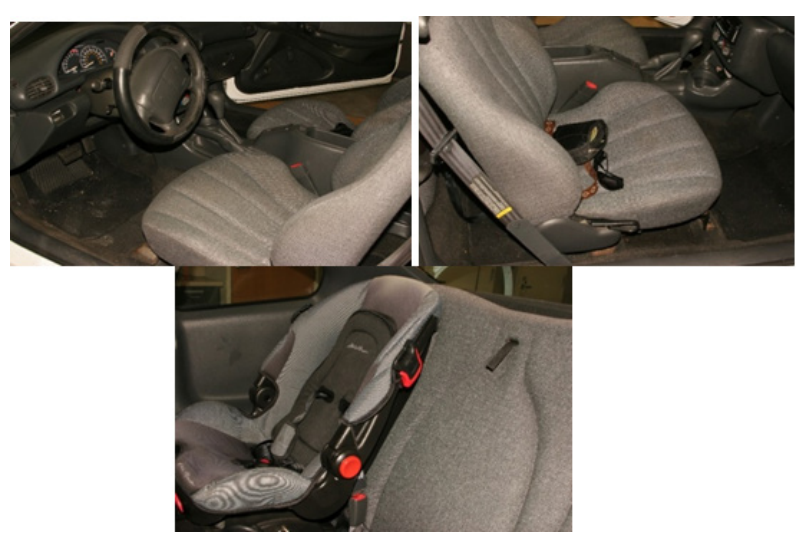


I inspected the interior using an Alternate Light Source and I observed two suspected stains on the interior topside of the vehicle. I performed a presumptive blood stain test on the stains. No reaction of blood was observed on the swabs.

\section{Inspection of the trunk area}

If the interior of the vehicle indicated suspected human decomposition, upon opening the trunk, my forensic experience confirmed at some point there was a dead body in this vehicle, as the smell in the trunk was three times greater. I observed a single hair strand on the trunk's edge (indicated by the orange identification pointer); and inside the trunk liner was some type of dirt residue and one dryer sheet freshener (indicated by the yellow letter placards). All of which were subsequently collected and removed, to include the spare tire, and taken to the forensic lab.

Case Presentation should provide detailed
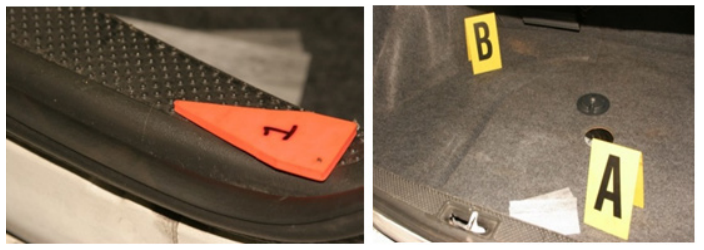

In the forensics lab I inspected the spare tire cover using an alternate light source and I observed suspected stain.

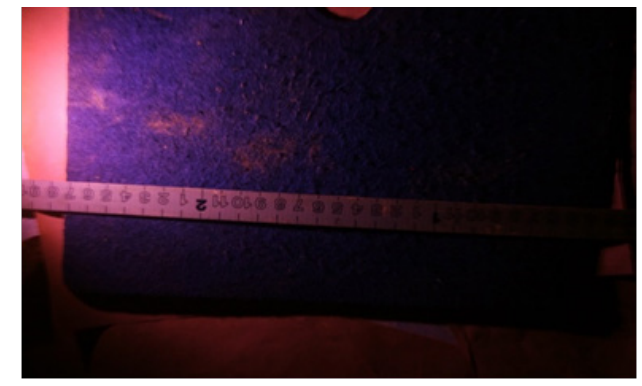

I began to apply what is called "blue star," on the spare tire cover with negative results to blood. I then took a swab sample from the suspected stain. I visually inspected the trunk liner and found several pieces of hairs, which were manually collected and preserved in a plastic petri dishes. Then I began to vacuum the vehicle beginning on the left side of the trunk liner. That was then placed in petri dishes and dirt and debris, as well as more hairs could be seen.

\section{Remove liner from the trunk of the vehicle}

As with the other items found in the trunk, the liner itself was also removed and transported to the forensics lab for further analysis.

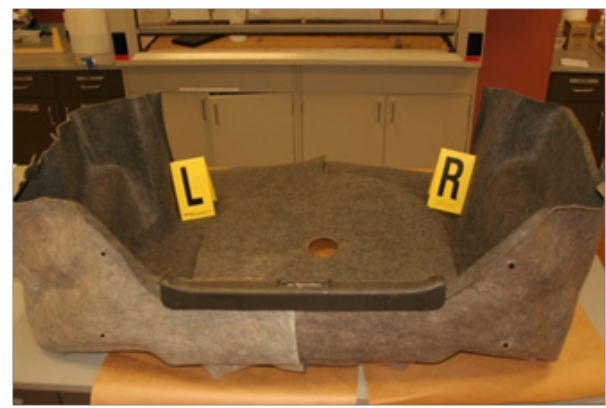

The collection of trace evidence, such as hair, is by far the most difficult evidence to locate, collect and preserve. It is akin to looking for a needle in a haystack. However, it is important to follow a process that would gain the best results to keep the evidence free from contaminates.

\section{Forensic tools and techniques}

1. Petri dishes

2. Metal / plastic tweeze

3. Post it ( sticky notes)

4. Magnifier with light

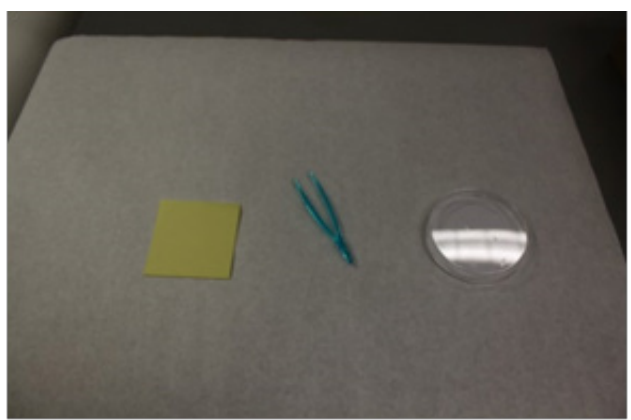

In this case I used two techniques for the searching and collection of hairs. Before beginning you must cover your hair and use face mask to prevent any of your own hairs from falling onto the item you are inspecting; and, because the hairs are so delicate, you don't want to breathe on them, therefore losing already fragile evidence. Once your gear is in place and your protectors on, you begin.

a) Magnifier with light. Once I locate the hair I immediately proceeded to mark the area where I locate the hair and photograph.

b) Use a tweezer to remove the hair and place the hair inside a petri dish.

c) The petri dish should be layered in a white paper, so when you place a hair in the dish, it is visible to the naked eye, and cover with lid to preserve hair(s).
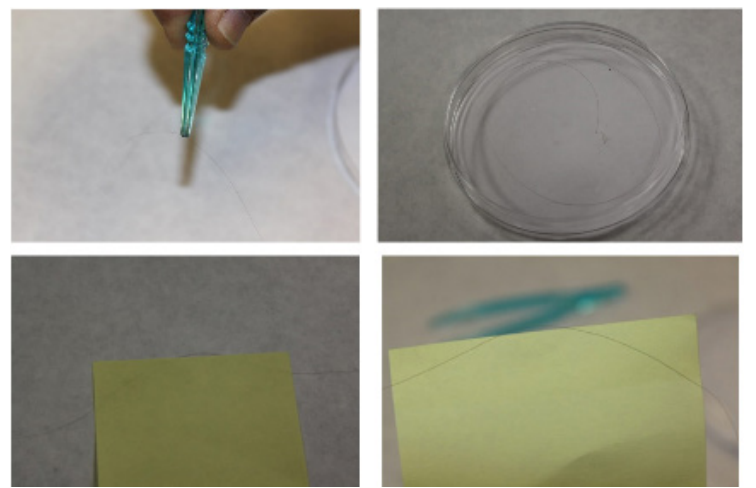

Another technique for the collection of hairs is using the adhesive backing of the post-it notes. It is a matter of preference and what the investigator feels more comfortable with using that will serve their purpose better. As for me, I prefer utilizing the adhesive of the post-it notes better, as it has a better feel to it. 
1. First locate, then photograph the hair, and afterwards you collect the hair utilizing the adhesive backing of the post-it note. (No preference to the color of the post-it notes).

2. Apply light pressure having the adhesive side over the hair, and the hair will attach itself to the adhesive side.

3. Once the hair is on the adhesive side, you fold the post-it note in half, once again utilizing the adhesive side to seal the hair inside the fold, keeping the hair secure. Place the post-note containing the hair into a yellow/evidence manila envelope. The following information should be on the front of the manila envelope:

4. A Case number,

5. Time and date,

6. Location of where evidence was found,Name of Crime Scene Investigator collecting said evidence.

7. All of which are then submitted to the FBI lab, to include, several pieces of the spare tire cover.

8. Place the post-note containing the hair into a yellow/evidence manila envelope. The following information should be on the front of the manila envelope:

9. Case number,

10. Time and date,

11. Location of where evidence was found,

12. Name of Crime Scene Investigator collecting said evidence.

All of which are then submitted to the FBI lab, to include, several pieces of the spare tire cover. Because of the evidence found, i.e. suspected human hairs, stains, and the possible odor of human decomposition, I must now consider this vehicle as "The mobile crime scene."

\section{The spun tale}

During this investigation, as it received nationwide attention, many know of the story Casey Anthony insisted to the Detective, along with her parents support, of Caylee being kidnapped by her nanny. However, based on the physical evidence found inside the trunk, that story being fed to detectives about what happened to Caylee, I knew to be untrue. Once again, my training experience is telling me the suspected odor, the stains and the hairs found in the trunk were that of a body being inside the trunk in a decomposing stage. Now, I had to ask myself two questions, even though my experience and gut feeling is telling me otherwise, I still had to ascertain that both the hair and the odor were from a human. So we bring out the big dogs, literally, we do bring dogs to the garage where the vehicle is being examined. Because K-9's are considered part of the Law Enforcement. Officer, we will refer to Gerus the K-9 as Officer Gerus, who is trained to detect the presence of human decomposition. Oftentimes, as part as a forensics investigation a k-9 is called into the investigation for the detection and verification for the presence of human decomposition. In this case, K-9 Officer Gerus examined the exterior of the vehicle and signaled to his handler the detection of human decomposition near the left rear quarter panel of the vehicle. This confirmed what K-9 Officer Gerus signaling to us is at some point, inside the trunk of this vehicle, a human body lay in a state of decomposition. Although
K-9 Officer Gerus confirmed my findings, still other questions arose, i.e. What measure can I take as a forensic investigator to prove our findings of the body in a state of decomposition in that trunk. All I know at this point is that I am on the right track, or better yet on the right scent.

\section{Oakridge national laboratory tennessee}

Dr. Apad Vass is a research scientist and forensic anthropologist at the life Sciences Division of Oak Ridge National Laboratory. Dr. Arpad Vass was therefore was called into the investigation to determine if there was any technique known that would be able to, verify or not, if the odor inside the trunk is that of human decomposition. Dr. Vass educated us on the use of collecting air sampling by using a device called ETS (Device that was used to collect the air samples)

\section{Summary of forensics evidence}

Over 600 pieces of evidence were submitted to the FBI Laboratory and several pieces of evidence were sent to Oakridge Laboratory in Tennessee. For the sake of brevity, I'll mention the most significant pieces of evidence in this case:

\section{First evidence}

The hair collected from inside the left side of the trunk liner of Casey Anthony's vehicle, which was submitted to the FBI lab. According to Karen Lowe, FBI Senior Analyst, the examination of the hair indicated an unusual dark band at a particular point near the root. The band on the hair occurs from that of a decomposed body. The hair contained mitochondrial DNA, better known as mtDNA. The mtDNA is passed down from the mother to the child with no contribution from the father, and the results of the mtDNA tests microscopically eliminated by length and color coming from Casey, Cindy or Lee. This indicated that the "death band" came from only one person, the missing child, Caylee Anthony. Thus changing the course of this investigation from rescue to recovery, from a missing child to a death investigation.

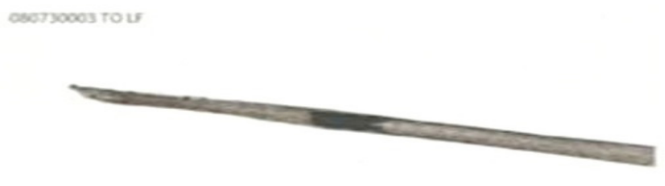

Note: This is the first time in the FBI Lab history a hair, in a questionable crime, was analyzed without body. This was one factor that completely changed the course of the investigation solving the question in reference to Caylee Anthony. To date, grandparents have sustained their granddaughter was kidnapped and is still alive.

\section{Second evidence}

Air samples (triple sorbent traps) from the trunk. This part of the collection was conducted by ASL Michael Vincent. The three triple sorbent traps were connected to TST pump which collected the air samples from the trunk. These traps are connected to LIBS (Laser Induced Breakdown Spectroscopy). 

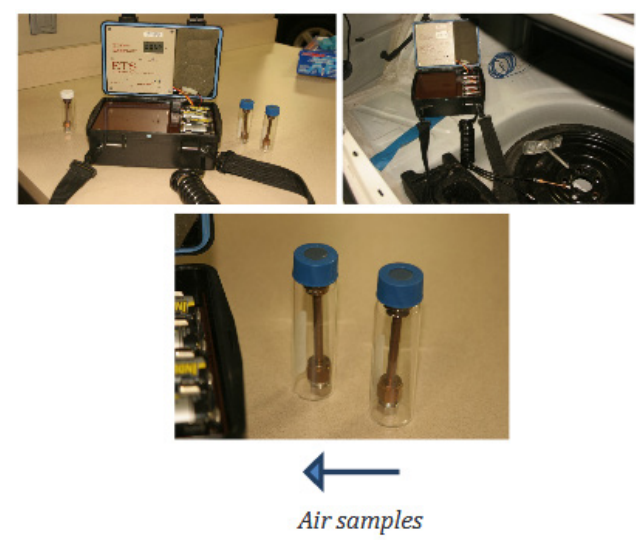

During the decomposition progression various organic elements are found in human tissues, i.e. magnesium $(\mathrm{Mg})$, calcium $(\mathrm{Ca})$, Iron $(\mathrm{Fe})$, and sodium $(\mathrm{Na})$, with increased concentrations (over time) in drainage (Figure 1). By using this technique we were able to determine these known inorganic components were elevated over the controls and also to determine if the concentration ratios of these elements could be used to determine a rough post-mortem interval, in this particular case (Figure 2).

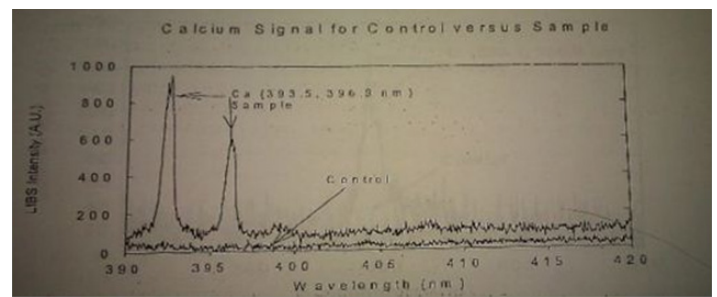

Figure I Spectra of control carpet and sample carpet for the presence of Calcium (Ca)

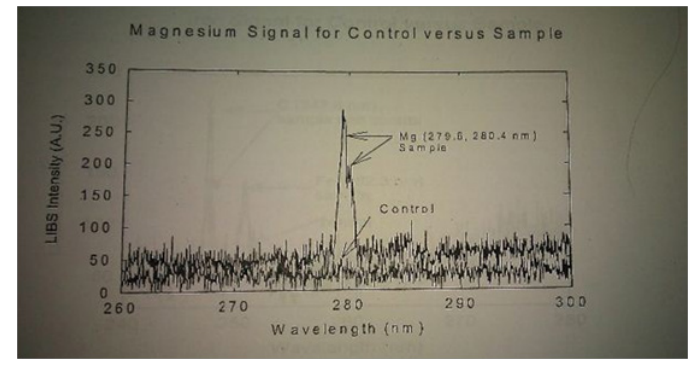

Figure 2 Spectra of control carpet and sample carpet for the presence of Magnesium (Mg).

\section{Result from oak ridge national laboratory}

Indicated a great portion of the total odor signatures identified in the trunk of the Casey Anthony vehicle were consistent with an event originating from that of human decomposition

\section{Conclusion}

Based on the first piece of evidence, the hair found in the trunk of a vehicle belonging to Casey Marie Anthony, which was determined to be coming from a decomposing human body. And that through mtDNA was that belonging to Caylee Marie Anthony, linked through the mother Casey Marie Anthony. Secondly, the air samples collected from the trunk which showed various inorganic elements found in human tissues during the decomposition process of a human, proved that Caylee Marie Anthony's body was inside the trunk of the vehicle. Because of that crucial evidence, was the grounds utilized to file criminal charges against Casey Marie Anthony. However, before said evidence could be used in the trial as being reliable and scientific, it first must pass the Frye hearing. This hearing consists of determining through the Frye standard, Frye test or general acceptance as reliable and scientific evidence. It provides that expert opinion based on a scientific technique is admissible only where the technique is generally accepted as reliable in the relevant scientific community. As such was the case in Daubert v Merrle Dow Pharmaceuticals 509 U.S. 579. ${ }^{4}$

\section{Acknowledgments}

None.

\section{Conflicts of interest}

None. 